Original Research Paper

\title{
Detection and Diagnostic Approach of COVID-19 Based on Cough Sound Analysis
}

\author{
${ }^{1}$ Muzhir Shaban Al-Ani, ${ }^{2 *}$ Thabit Sultan Mohammed, ${ }^{3}$ Awni Ismail Sultan, ${ }^{4}$ Hasan Ismail Sultan, \\ ${ }^{5}$ Khattab M. Ali Alheeti and ${ }^{2}$ Karim Mohammed Aljebory \\ ${ }^{1}$ Department of IT, College of Science and Technology, University of Human Development, Sulaymaniyah, Iraq \\ ${ }^{2}$ Department of Computer Technical Engineering, Al-Qalam University College, Kirkuk, Iraq \\ ${ }^{3}$ Department of Surgery, College of Medicine, Tikrit University, Tikrit, Iraq \\ ${ }^{4}$ Department of Internal Medicine, College of Medicine, Tikrit University, Tikrit, Iraq \\ ${ }^{5}$ College of Computers and Information Technology, University of Anbar, Anbar, Iraq
}

\section{Article history}

Received: 25-03-2021

Revised: 04-06-2021

Accepted: 15-06-2021

Corresponding Author: Thabit Sultan Mohammed Department of Computer Technical Engineering, AlQalam University College, Kirkuk, Iraq

Email: thabit.tsm@gmail.com

\begin{abstract}
Coronavirus (COVID-19) started at the end of 2019 and then spread out around the world as a pandemic at the beginning of 2020. At that time, researchers began to work on detecting and diagnosing this virus, where many methods have been applied for this reason. This study focuses on how to diagnose coronavirus through patients' cough. Accordingly, real samples were taken from people infected by the coronavirus and others, who are suffering from some respiratory diseases. The cough of a person with coronavirus is characterized by its dryness and differs from other cough sounds through a set of factors that are considered for study and analysis through this study. Among these factors is the sound energy, which is found to be the most effective factor and hence implemented as a key indicator for COVID-19 detection. The discrete wavelet transform is the adopted method to realize the detection process via approximation and the analysis of coefficients details. The obtained results show acceptable detection accuracy for the considered samples. Minor mismatching in the detection process is noticed during the procedure, which is mainly due to some patients being infected with the respiratory diseases that exhibit similar symptoms.
\end{abstract}

Keywords: Corona Virus, COVID-19, Cough Sound, Signal Processing, Statistical Analysis, Feature Extraction

\section{Introduction}

Due to the rapid spread from one individual to another in the society, the new coronavirus (also called COVID-19) has resulted in a global prevalent problem, where the World Health Organization (WHO) on January 30, 2020 declared this disease as an epidemic. Moreover, the great increase in case numbers has led to the exhaustion of health care systems in many countries (Tabata et al., 2020). The coronaviruses formerly detected could only be the head of the iceberg of the total infection, with potentially further novel and serious zoonotic events to be discovered. Furthermore, there are noticeable epidemiological variations between Asian and European COVID 19 infection concerning prevalence rate of the disease and the fatality (Sultan et al., 2020).

Coronaviruses are enveloped non segmented positive sensing RNA viruses beyond the family of Coronaviridae, they widely infected humans as well as other mammals (Huang et al., 2020). They are so termed because their similar shape to the sun's corona through the total solar eclipse, where they have a fringe of large, bulbous surface projections (Holland et al., 2020).

The virus responsible for the present outbreak of infection was primarily named novel corona virus, now renamed as SARS COV2 or COVID 19. Additionally, it is associated with Severe Acute Respiratory Syndrome Coronavirus (SARS CoV) and Middle East Respiratory Syndrome Coronavirus (MERS CoV), but COVID 19 infection can result in potentially fatal consequences among susceptible individuals (WHO, 2020a; Xavier et al., 2020). Moreover, the spread of COVID-19 is rapid. Transmission can occur directly during patient coughing, sneezing or talking, which produces infected droplets that can contaminate the mouth, nose or eyes of another individual or indirectly by touch the surfaces in the immediate environment or with objects used on or by the infected patients, causing infection (WHO, 2020b). 
COVID 19 connects strongly to AngiotensinConverting-Enzyme- 2 (ACE2) cellular receptors in the host cells through the spiky progressions of glycoprotein on the viral envelope. These receptors are located on the surface of the lung's alveolar cells, intestine, kidneys, heart, neurons, nasal and oral epithelial cells. Therefore, active viral replication may induce damage to these organs, present as a mild symptoms or progress to severe respiratory failure with multiple organ failure (Hoang et al., 2020; Ni et al., 2020).

Incubation period after exposure to infection can range from 2 to 14 days. The common presenting symptoms are fever, chills, cough, fatigue, myalgia, anorexia and dyspnea (Wang et al., 2020). These may be accompanied by respiratory secretions, hemoptysis, diarrhea and headache. Loss of smelling (anosmia) and taste loss (ageusia) are common symptoms and considered as a screening tool for possible COVID 19 infection (Mehraeen et al., 2020; Agyeman et al., 2020). Furthermore, about $5 \%$ of patients develop one or more of the following complications: Acute Respiratory Distress Syndrome (ARDS), respiratory failure, septicemia and septic shock, coagulation disorders and thromboembolism and/or multiple organ failure such as liver failure, acute renal failure, heart failure, cardiogenic shock, myocarditis, or cerebrovascular accident (PAHO/WHO, 2020). Indeed, mortality is significant in the elderly and those with comorbidities as heart diseases, hypertension, diabetes, chronic respiratory diseases and malignancy (Wang et al., 2020).

However, the recognition of COVID 19 nucleic acid by Reverse-Transcription-Polymerase-Chain-Reaction (RT PCR) is considered the gold standard for viral identification in patients with clinical symptoms with very high sensitivity (>95\% over numerous studies). Chest Computed Tomography (Chest CT) is a quick test to perform and may help in the diagnosis of COVID 19, particularly in the setting of resource limitation (Xavier et al., 2020; Velavan and Meyer, 2020; Young et al., 2020).

Until currently available information, there is no treatment that is precisely considered for coronavirus infection except for early diagnosis, isolation and supportive treatments for affected patients. Therefore, maintaining of hand hygiene, keeping a distance of at least 6 feet away from the patient and use of medical masks are effective preventive measures in the outpatient healthcare setting (Wang et al., 2020).

Great amount of efforts are employed to develop highly accurate diagnostic testing for COVID-19 (Sheridan, 2020). Validation of new tests from different manufactures across the globe is a major concern of the relevant health international organizations (Cheng et al., 2020). Different primary technologies are used to test for COVID-19 infection, in addition to new technologies that have the potential to accelerate the coronavirus detection and diagnosis, including digital PCR, CRISPR and microarray (Xu et al., 2020).

Different scientific and engineering techniques are adopted by researchers to diagnose COVID-19 including, soft computing techniques and practices such as; neural networks, fuzzy logic and genetic, as well as signal and image processing. The power behind most of these techniques is their support to decision making.

Coughing, which is the early recognizable symptom of COVID-19, is basically a sound that can be represented as an audio signal and hence studied to help in the diagnosis process. Signal processing along with soft computing techniques may be applied on the cough sound signal for the purpose of analysis and study.

In this study, we have adopted the collection of cough recordings from a society of patients who are divided up into two basic groups; namely patients diagnosed as COVID-19 infected and a group of patients who are not COVID-19 infected. An algorithm has been developed to use these cough sounds as input signals for processing. Effective features of the studied signal are selected for analysis. Statistical calculations in addition to the use of neural network are implemented to help in the final decision about the detection and diagnosis of the disease.

In the next section of this study, a literature review is presented with the most relevant researches. Section 3, states the nature of data used in the study and the way by which the patients involved, are grouped. The proposed algorithm and the application concerning calculations and analysis of its individual steps are included in section 4. Section 5 includes the results and their related discussion. Finally, section 6 is presenting the concluding remarks about the paper.

\section{Related Work}

Medical literature shows that cough patterns are linked with changes in anatomy and physiology of the respiratory system (HuBer and Stathopoulos, 2015). This fact has always been a motivation for researchers to propose methods for diagnosis using speech and acoustics research and relying on study and analysis of dataset of respiratory sounds. In this section, we present related work and proposed methods that considers the analysis and identification of cough as a base for diagnosis. It's possible to conclude that the goal behind research in this field is not to produce replacement for the existing chemical testing practices but to supplement them with techniques that are cost effective, fast and simpler.

A method for quantitatively analyzing cough sound was proposed and described in (Thorpe et al., 2001). In their research, used cough samples, that were collected from normal adults before and after meth choline. Cough sound in this study is represented by a set of spectral and temporal features. The results show that even with the 
existence of some inconsistency of the considered features, it is still able to characterize significant differences in the cough sound after meth choline challenge, which leads to a conclusion that sound processing and analysis of the cough is able to provide information that could be useful in diagnosis.

Chatrzarrin et al. (2011), proposed two features for the analysis of cough sounds and extract effective features that can discriminate between cough sounds being dry or wet. The features considered are the number of peaks of the energy envelope and the power ratio of two frequency bands of the second phase of the cough signal.

Song (2015), proposed a cell phone-based low-cost and rapid diagnosis method for respiratory health problems. Targeted patients were grouped based on their ages and features were selected for each age group using the analysis of variance for a large set of acoustic features. The model of Song was assessed based on the fact that given features representing the illness pneumonia is either found or not. Authors claimed that an effective classification is obtained even in the presence of environmental noises.

A general outline for cough sound analysis was presented by Infante et al. (2017). The work considered cough segmentation, feature extraction and design of a general classification. Four features were selected (variance, kurtosis, rate of decay and zero crossing irregularity). A set of cough data collected from 87 persons, where some of them were patients presenting a combination of pulmonary conditions, as well as healthy individuals.

You et al. (2017) considered the use of pattern recognition technologies to offer clinical information for cough diagnosis and monitoring towards the evaluation of the intensity and quantity of cough based on cough detection. It was recorded in this research that the energy spectrum of cough signal is different from a speech signal, where it widely spreads in the whole frequency band. Additionally, the spectral structure from cough signal can be used as filter banks of feature extraction methods. Filter banks were made more suitable for cough detection than manually designed ones by using this idea.

Early identification of coughs was the main motivation of the research by Pramono et al. (2019). This work stated that the management of certain diseases such as asthma and COPD is highly improved through cough identification because it is a common symptom of such respiratory diseases. An algorithm based on four features was presented to automatically identify cough events from acoustic signals. It was mentioned that the proposed algorithm demonstrated good performance in spite of its small size of feature-space.

Miranda et al. (2019), the authors proposed that a key to track the condition of patients suffering from Tuberculosis (TB) can be achieved via cough detection and identification. Several acoustic features were adopted to perform cough detection. Among such features are the Short-Time Fourier Transform (STFT), Mel Frequency Cepstral Coefficients (MFCC) and Mel-scaled Filter Banks (MFB). Deep neural networks and convolutional neural networks architectures were used to evaluate the features.

Soliński et al. (2020) presented a model for automatic cough identification based on airflow signals. The proposed algorithm was trained and validated by a set of data obtained from a web application called AioCare. The database of spirometry curves from the NHANES database by the American National Center for Health Statistics was also used. After the evaluation of many classifiers, the feed-forward artificial neural network was selected as the ultimate classifier.

Rudraraju et al. (2020) stated that the commonness of respiratory diseases in the world as a whole and specifically in rural areas, is due to the lack of methods that are cost-effective for early diagnosis. The analysis of cough sounds can help to extract vital information about the respiratory system. Therefore, authors of this research proposed that a blend of standard signal processing features and domain-specific features show a crucial role in cough patterns. Such patterns include narrowed airway, widened airway, stiff lungs and fluid filled air sacs.

Shi et al. (2018) presented a review that surveyed the principles of cough sound generation, related clinical studies and an analytical study about some successful cough monitoring equipment and their recognition algorithms.

Abeyratne et al. (2013) stated that 1,800,000 children throughout the world annually killed due to Pneumonia. The vast majority of these losses occur in remote regions, where the lack of field-deployable imaging and laboratory facilities together with the shortage of competent healthcare workers considered as the main causes. Researchers proposed a system for automated analysis of cough and respiratory sounds. They have assumed that cough carries vital information to diagnose pneumonia and hence collected cough sounds and extracted features such as non-Gaussianity and Mel Cepstra and used them to train a Logistic Regression classifier.

Barry et al. (2006) developed a program based on digital signal processing used for the analysis of digital audio recordings. It was designed to calculate the characteristic and spectral coefficients of sound events. Since cough is a sound, therefore, the system that is adopting Probabilistic Neural Network (PNN) can be used to calculate the over-all number of coughs and cough frequency as a function of time.

Porter et al. (2019), it was stated that, respiratory disorders represent a significant global disease burden. Diagnosis of these diseases can be challenging even for experienced clinicians with access to diagnostic support services. A method to diagnose pneumonia based on the automated analysis of cough sounds were, therefore proposed. The proposed method, that can help in early 
and more accurate diagnosis, may only require minimal operator training. The technology used is similar to that adopted for speech recognition.

Shi et al. (2020) presented a review paper with a summary of the latest research findings with concentration on the efforts and experience in China. Recent research has revealed critical aspects of COVID-19 biology and disease pathogenesis. Other studies have focused on epidemiology, clinical features, diagnosis, management, drug and vaccine development. A vision on how to contain and improve the understanding of the infectious and widely spreading disease, together with updated guidance for prevention, control and critical management of this pandemic.

Sharma et al. (2020) mentioned that a need for diagnosis methods for COVID-19, in addition to the (RT-PCR) testing, are necessary. They proposed the use of machine learning techniques for the analysis of cough sounds. In their work on progress, an open-source database named Coswara was established with sound samples collected through worldwide crowdsourcing using a website application.

Brown et al. (2020) used a dataset of respiratory sounds that is collected to aid diagnosis of COVID-19. Coughs and breathing sounds were used to understand how to differentiate COVID-19 sounds from those in asthma or healthy controls. This work was claimed to achieve promising performance and still open for further investigation of how to automatically analyze respiratory patterns and use results as pre-screening signals to aid COVID-19 diagnosis.

The COVID-19-RCC protocol, presented by Derraz (2020), is an algorithm based on the analysis of the audio frequency spectrum during phone calls. During the phone's conversation, which is not recorded, the cough is recognized and the count of coughs is recorded. Analysis of the detected cough, can predict the stage of illness and the probability of infection by transferring the virus to people in the surrounding area. In order to limit the spread of the virus, detecting patients who complain of coughing is a priority, where coughing is the main factor behind the droplets.

A smartphone application, named AI4COVID-19 was proposed by Imran et al. (2020). In this application, which is a cough-based diagnosis tool of respiratory diseases, a recording of three $3 \mathrm{sec}$ cough sound is sent to an AI engine running in the cloud, which then returns a result within 2 min. Though the AI4COVID-19 system is a work on progress, its accuracy is claimed promising enough to encourage a large-scale collection of labeled cough data. The AI4COVID-19 system is not at the level to be considered as a clinical grade testing tool. Instead, it offers clinical decision assistance to those who need it the most, thereby saving more lives.

\section{Data Collection and Specification}

A prospective study includes 2 groups of patients, who attend the main primary health care center in AlZab district.

COVID-19 patients' group: COVID-19 infection was confirmed by PCR testing (from nasopharyngeal and oropharyngeal swabs) in Public Health Laboratory in Kirkuk.

Non-COVID-19 patients' group: Patients who complain from other respiratory problems like asthma or chronic obstructive pulmonary disease.

Cough sounds were recorded by using (HONOR 8x Smartphone). All patients asked to take deep breath and cough through their mouth.

The study was accepted by ethical committee of College of Medicine-Tikrit University and Kirkuk directorate of health in Iraq. All patients were instructed about the study and their agreements were taken.

\section{Proposed Approach of COVID-19 Detection and Diagnosis}

\section{Cough Sound Characteristics}

A sudden and acute expulsion of air from the lungs that acts as a preventive mechanism to clear the airways or as an indication of a lung disorder. Cough sound is generally divided into three impacts (parts) as shown in Fig. 1:

- First impact, explosive expiration via suddenly opening of glottis

- Intermediate impact, attenuation of cough sounds

- Third impact, the voiced phase via closing of the vocal cord

In addition, there are a variety of cough patterns and some cough sounds only have two parts. Coughing can be measured in the following ways:

- Sounds of explosive cough indicating the number of explosive cough impulses

- Seconds of cough indicating the period of an explosive part

- Breaths of cough indicating the respiration rates including a cough

- Epochs of cough indicating the number of cough sounds within two seconds

The intensity of cough can be measured by sound power, peak energy and mean energy and these indexes can be calculated for a time from the explosive cough sound.

Detection and Diagnostic Approach Based on Cough Sound Analysis

There are many techniques existing in literature and applications for the cough detection. While most of these 
existing techniques are based on adopting the medical diagnosis principles, our research is, however, focusing on the design and implementation of an efficient approach for diagnosis and detection of COVID-19 disease via extraction and analysis of the cough sound characteristics.

The steps represent the procedure that our approach is adopting, are shown in Fig. 2.

Acquisition of the cough sound: The cough sound is basically a form of voice signal. It is captured by a specific microphone that convert the voice vibrations into electrical voice signal, which is received by a computer device as a digital voice signal.
Preprocessing: The preprocessing sub steps include, setting the frame length calculation to be 20000 samples, sampling frequency calculation, signal length resizing and noise removal. Additionally, a median filter is applied to smooth the signal and remove the unwanted parts from it. The signal to noise ratio is increased leading to the signal characteristics, to be measured, being improved.

Silence removal: In order to get real voice signal, it is important to remove the silent parts of the signal, while keeping the cough sound. Silence removal is achieved by tracking the start and end of cough events depending on thres holding according to the features of time domain.

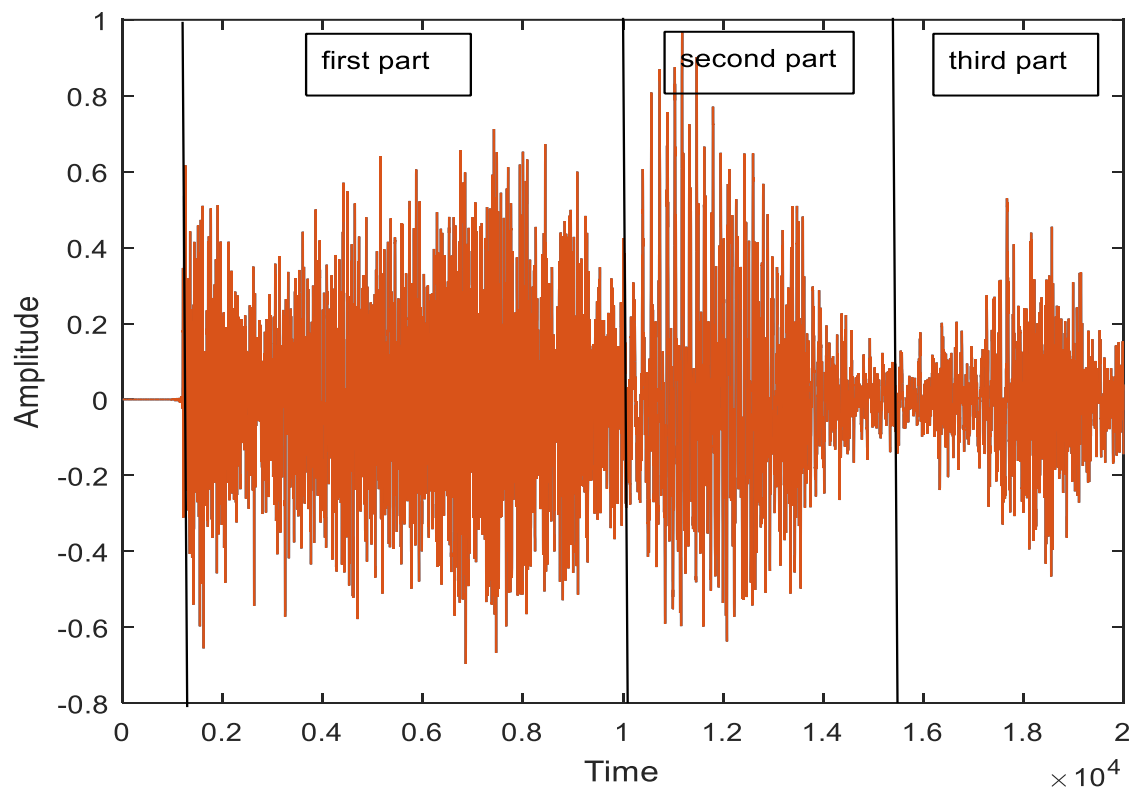

Fig. 1: Parts of cough sound
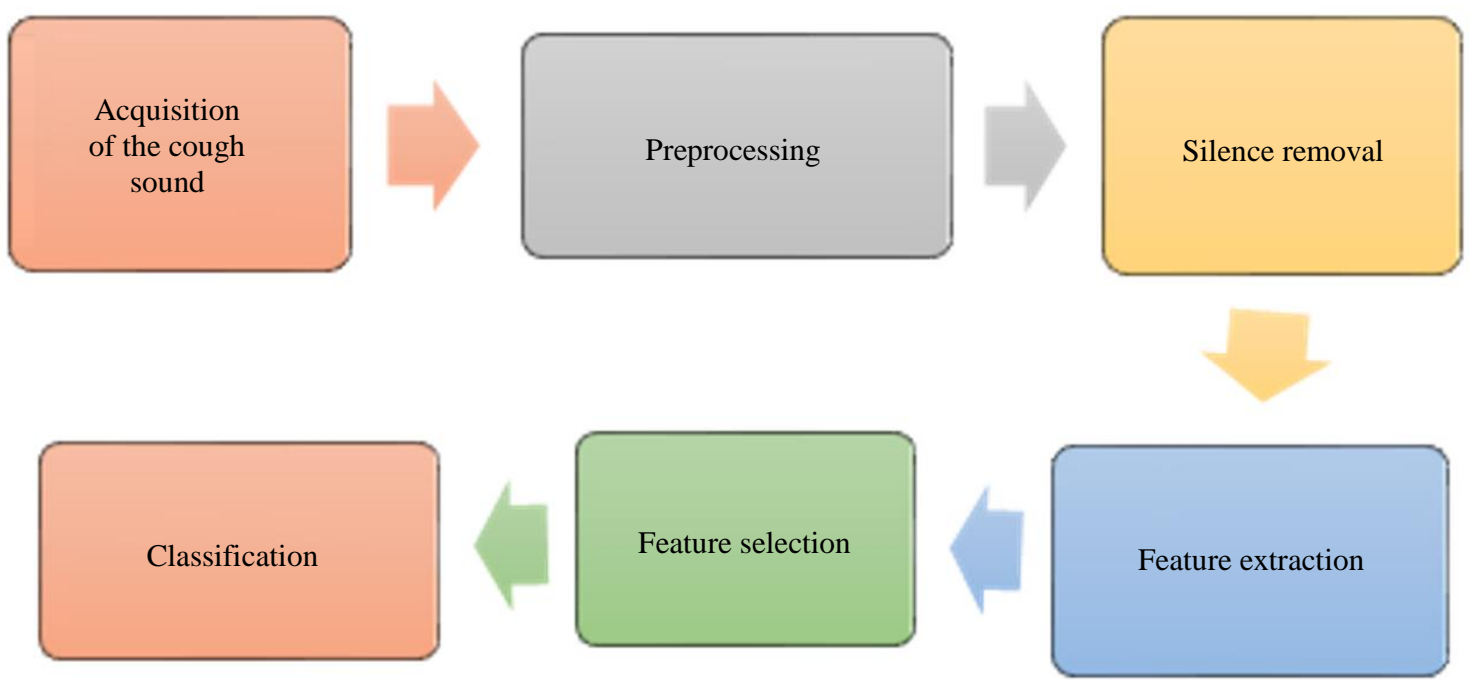

Fig. 2: Cough sound analysis for detection and diagnosis 
Feature extraction: Extracting features from our cough sound signal is performed by applying one dimensional Discrete Wavelet Transform (DWT) (Wali et al., 2012). The one-dimensional discrete wavelet transform is a combination of low pass filter and high pass filter passing through the voice signal in order to generate approximation coefficients for low pass filter and details coefficients for high pass filter as shown in the following equation (Alheeti et al., 2019):

$$
|A[i, j] D[i, j]|=(x * g)[i]=\sum_{j=1}^{N} x[j] g[i-j]
$$

where, $x[i]$ represent the input voice signal and $g[i]$ represents the mask elements.

Feature selection: In this step, the most relevant and effective features that are used in the classification are selected.

Energy (E): Two important factors are calculated which are the percentage of energy corresponding to the approximation $E_{a p p}$ and the vector containing the percentages of energy corresponding to the details $E_{d e t}$ (Rocchesso, 2003):

$$
\begin{gathered}
E_{\text {app }}[i]=\sum_{j=1}^{N}|A[i, j]|^{2} 1 \leq i \leq N \\
E_{\text {det }}[i]=\sum_{j=1}^{N}|D[i, j]|^{2} 1 \leq i \leq N
\end{gathered}
$$

Zero-Crossing Rate (ZCR): This rate indicates the number of times a signal crosses zero amplitude giving a reference to the audio signal frequency. High value of ZCR related to high frequency audio signal and visa-versa:

$$
Z C R=N Z C * \frac{F_{s}}{N}
$$

where, $N$ represents number of samples per frame and $F_{s}$ is the sampling frequency.

Number of Zero Crossing (NZC) is given by the following equation:

$$
N Z C_{i}=\sum_{n=1}^{N} \operatorname{sign}\left[x_{i}(n)\right]-\operatorname{sign}\left[x_{i}(n-1)\right]
$$

where, $\operatorname{sign}[x]=\left\{\begin{array}{c}1 \text { if } x \geq 0 \\ -1 \text { if } x<0\end{array}\right\}$

In the implemented approach, a median filter is added in order to reduce the noise as much as possible, which exhibits a noticeable purification to the cough signal.
Skewness(SK): Is the third standardized moment and it indicate the asymmetry of a symmetric probability distribution, where it refers to distortion in a symmetrical distribution of data (DeCarlo, 1997). Skewness is given by the following equation:

$S K=\frac{\frac{1}{N} \sum_{i=1}^{N}\left(x_{i}-\mu\right)^{3}}{\frac{1}{N} \sum_{i=1}^{N}\left(x_{i}-\mu\right)^{3 / 2}}$

where, $\mu$ represents the mean value.

There are two types of skewness: A positive skewness occurs when mean and median are greater than the mode and negative skewness occurs when mean and median are less than the mode.

$\operatorname{Kurtosis}(\mathbf{K U})$ : Represents a measure of whether the data are heavy-tailed or light-tailed relative to a normal distribution. It is given by the following equation (Mohammed et al., 2020):

$K U=\frac{\frac{1}{N} \sum_{i=1}^{N}\left(x_{i}-\mu\right)^{4}}{\left(\frac{1}{N} \sum_{i=1}^{N}\left(x_{i}-\mu\right)^{2}\right)^{2}}-3$

A high kurtosis indicates that data has heavy outliers and low kurtosis indicates that data has lack of outliers.

Kaiser filter: THIS filter, in addition to SavitzkyGolay filter, are used here in order to remove the maximum passible unwanted noise. In this filter, the Kaiser window is calculated as per the following equation (Al-Ani, 2017):

$w(i)=\frac{I_{o}\left(\beta \sqrt{1-\left(\frac{1-N / 2}{N / 2}\right)^{2}}\right)}{I_{o}(\beta)} 1 \leq i \leq N$

where, $I_{0}$ is the zeroth-order modified Bessel function and $N$ is the number of points:

$\beta=\left\{\begin{array}{l}0.1102(\alpha-8.7), \alpha>50 \\ 0.5842(\alpha-21)^{0.4}+0.07886(\alpha-21) \quad 50 \geq \alpha \geq 21 \\ 0, \alpha<21\end{array}\right\}$ (9)

where, $\alpha$ side lobe attenuation

Savitzky-Golay filter: It is a Finite Impulse Response (FIR) smoothing filter of polynomial order, using a 2nd order polynomial and 7 data points (Al-Shayea and Al-Ani, 2016): 


$$
\begin{aligned}
& y_{i}=\left(-2 x_{i-3}+3 x_{i-2}+6 x_{i-1}+7 x_{i}\right. \\
& \left.+6 x_{i+1}+3 x_{i+2}-2 x_{i+3}\right) / 2
\end{aligned}
$$

Classification: In the classification stage, the voice samples that have already passed through voice preprocessing, silence removal, feature extraction and feature selection will go through a decision stage by which the samples are classified into groups based on certain rules.

\section{Results and Discussion}

Cough sound collections and their relevant data that are used for his study were conducted in the main primary health care center at Al-Zab district, Iraq. Two groups of patients are included; COVID-19 patients' group (positive infection with COVID-19) and Non-COVID-19 patients' group (negative infection with COVID-19, however they are infected by other respiratory diseases). The implementation of the proposed approach to detect and diagnose the COVID-19 disease based on the analysis of cough sound passes into many steps starting from preprocessing and ending with decision making to classify them as negative and positive cases.

\section{Selected Features Based Analysis}

An example of the acquired original cough sound signals is shown in Fig. 3.
In part (a) of Fig. 3, a case of a patient diagnosed as not being infected with corona virus (Negative case), while part (b) belongs to a positive case. In part (a), wide band of frames are shown, whereas condense occurrence of samples within the frame are shown in part (b).

A single frame of cough is selected in the next stage based on criteria considering the proper threshold. The implemented frame selection of cough sound signals is shown in Fig. 4.

Since the patients' cough sounds were recorded using a regular cellphone device, there expected to be a noticeable amount of noise. Reduction of this added noise is highly recommended; therefore, two types of filters are applied, namely Kaiser and Savitzky-Golay filters.

In Fig. 5, the application of these filters on a noncovid-19 case cough sound signal and a Covid-19 case are demonstrated in parts (a) and (b) respectively.

During the analysis of the cough sound signals, we have found that the power of the signals is an effective feature to characterize induvial signals. Therefore, this feature is being selected and adopted as a key feature helping in the process of signal classification and hence diagnosis of infection. Figure 6 ( $a$ and $b$ ) is illustrating periodogram functions for a negative (non-COVID-19) case and a positive (COVID-19) case respectively. A clear difference between the two cases can be noticed in the figures. Furthermore, the energy Eq. 2 and 3, mentioned above, are also used in the measurement of the signal power.

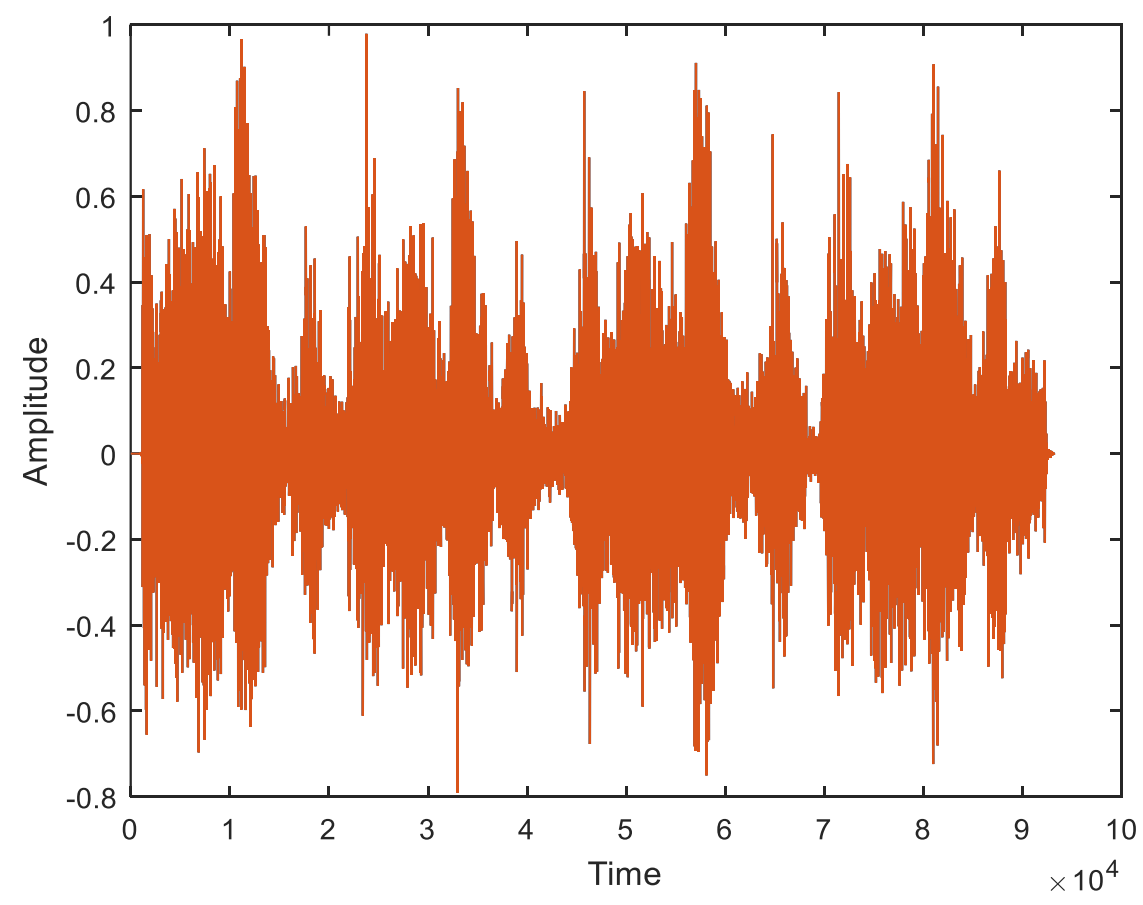

(a) 


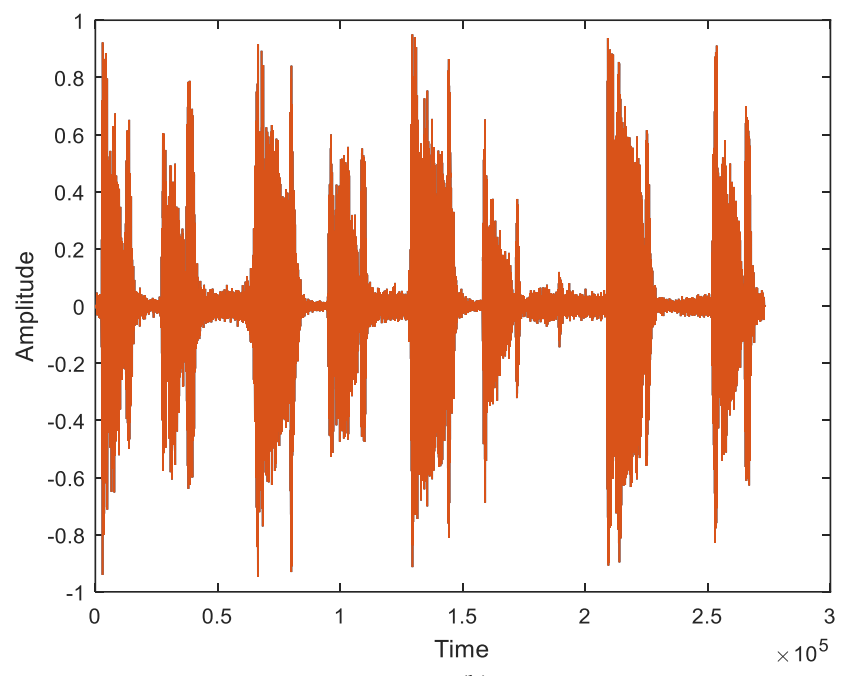

(b)

Fig. 3: Original cough sound signal (a) Negative case (non-COVID19); (b) Positive case (COVID-19)

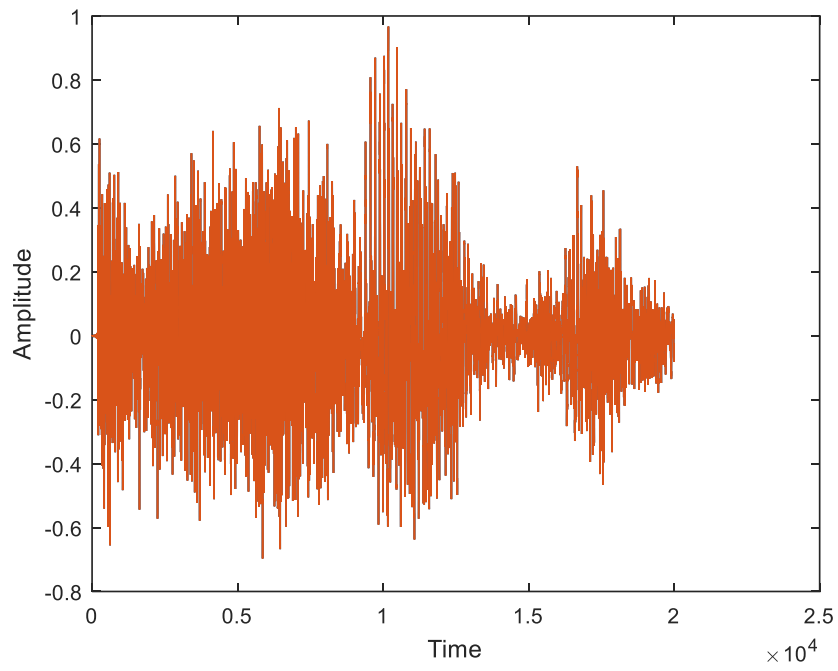

(a)

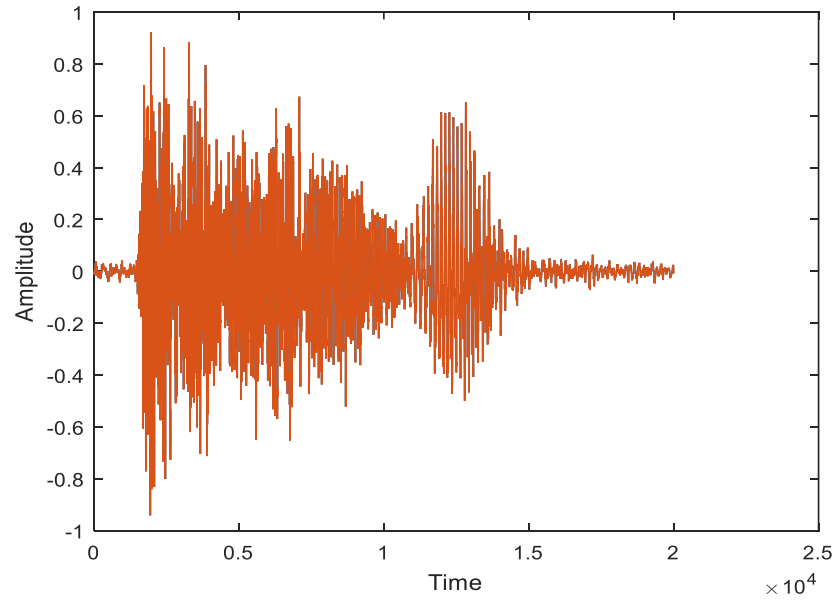

(b)

Fig. 4: Frame selection of cough sound signal (a) Negative case (non-COVID19); (b) Positive case (COVID-19) 

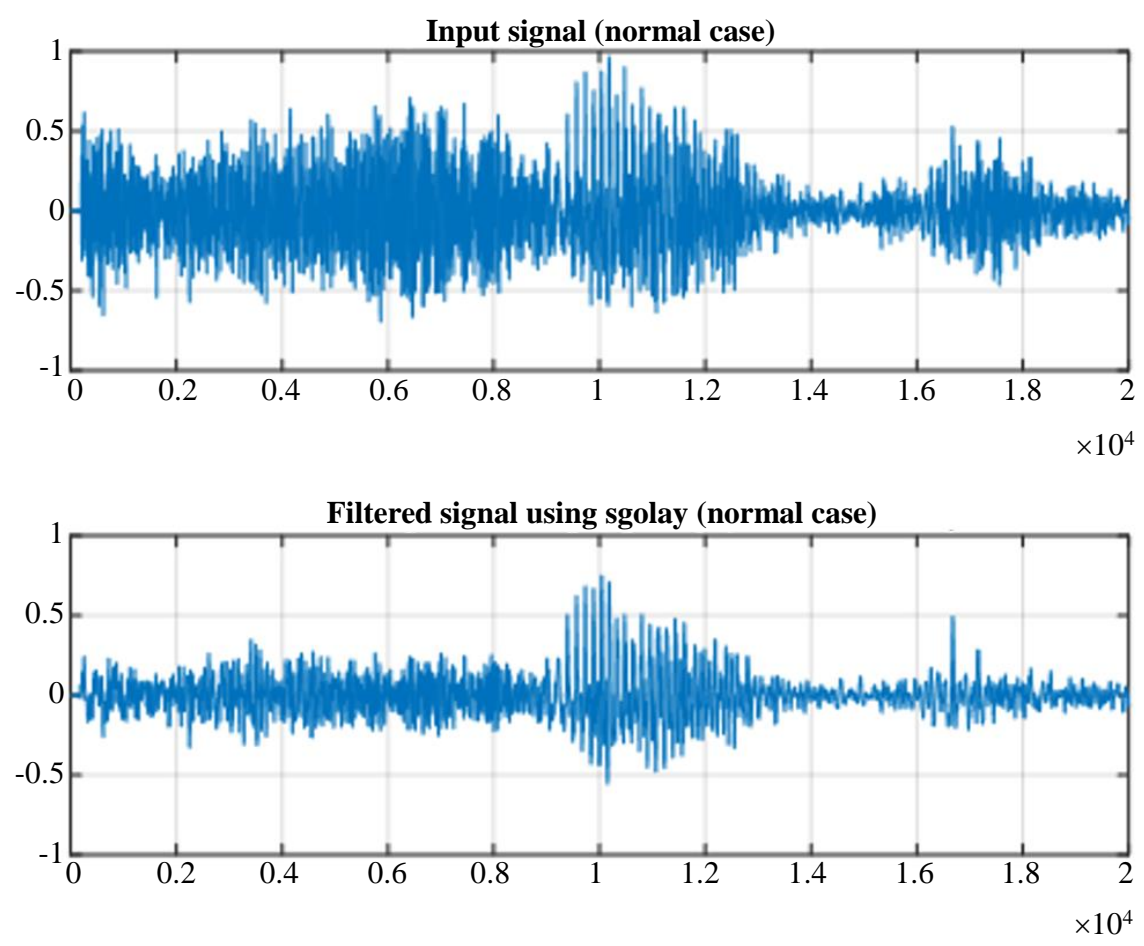

(a)
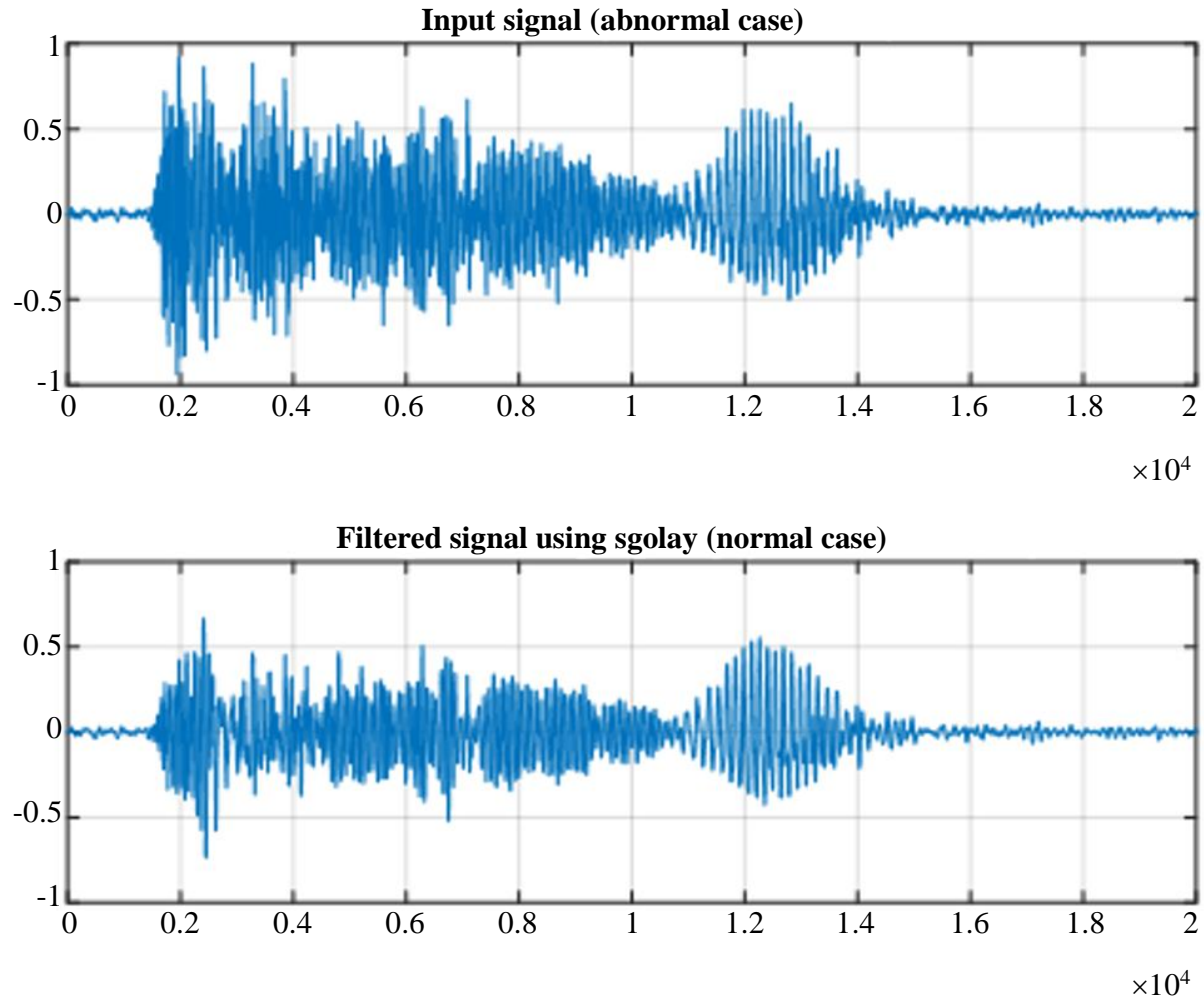

(b)

Fig. 5: Applying filter on cough sound signal (a) Negative case (non-COVID19); (b) Positive case (COVID-19) 


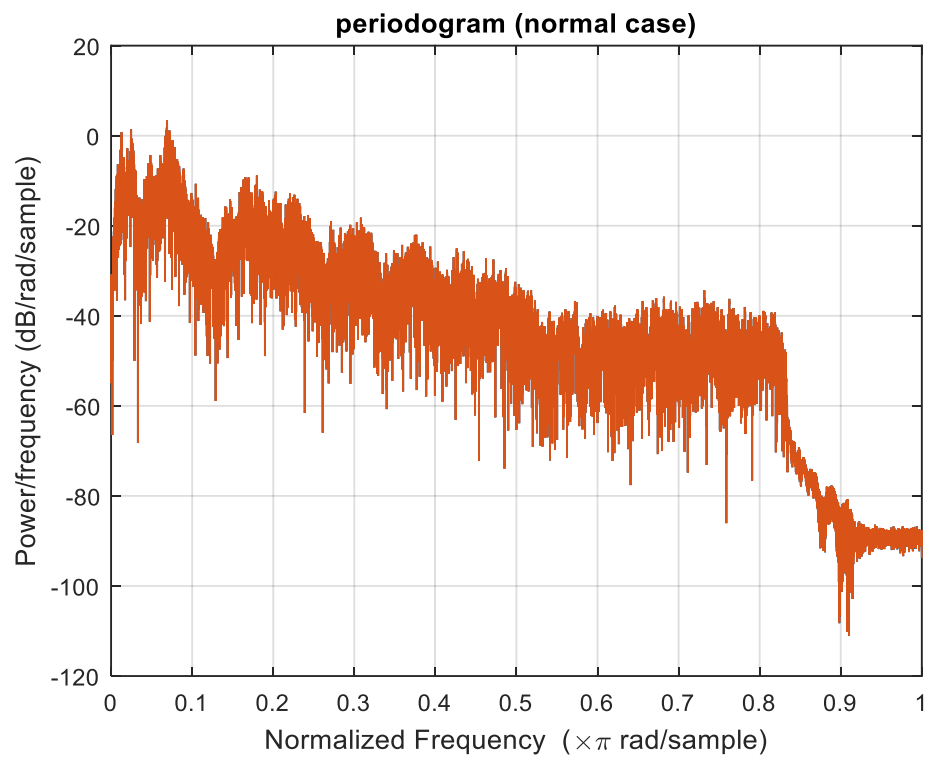

(a)

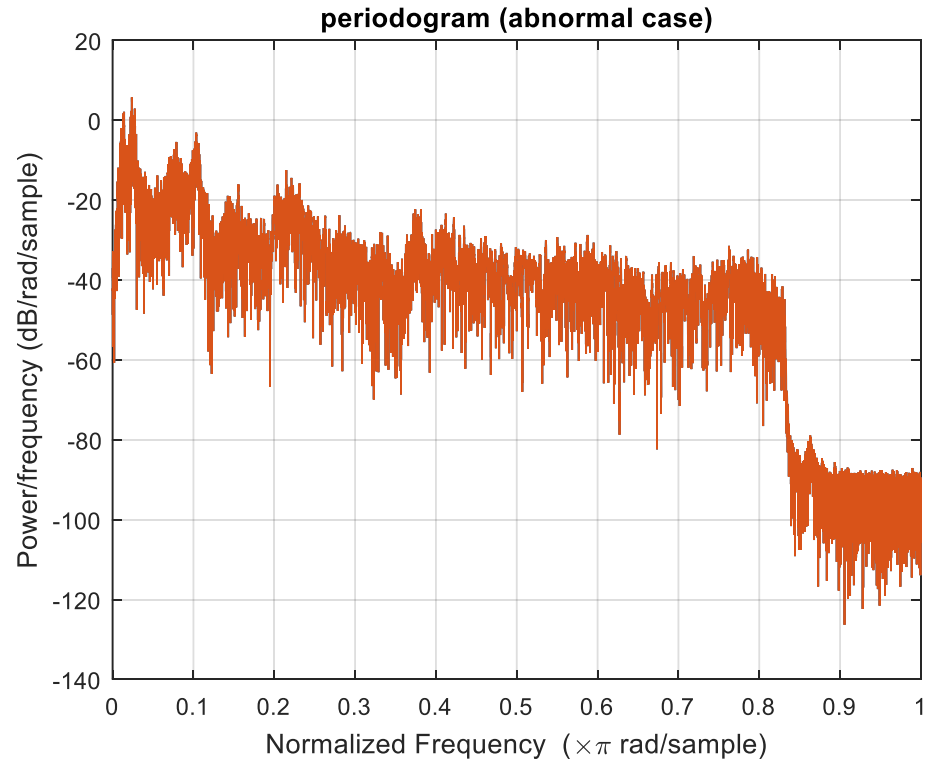

(b)

Fig. 6: Frame periodogram of cough sound signal (a) Negative case (non-COVID19); (b) Positive case (COVID-19)

The band spectrogram distribution in both cases is also considered in the analysis. Fig. 7, illustrate the change in frequency content over time. There are more interruptions in the positive (COVID-19) case spectrum and the amplitude of the frequency is not as homogenous as in the negative (non-COVID-19) case spectrum. Knowing that the darker frequencies are having higher amplitudes.

The Fourier spectrum of a signal is used to find the content of the frequency, whereas applying the discrete
Fourier transform on discrete-time signal is corresponding to time-frequency transform.

Mapping a digital signal $x(n)$ with a length $N$ into frequency domain $\mathrm{X}(\mathrm{k})$, can be represented as follows:

$$
X(k)=\sum_{n=0}^{N-1} x(n) e^{-j 2 \pi n k / N}
$$

The most common representation to visualize the spectra is used via log-spectrum, (log-spectrum = $\left.\log _{10}|\mathrm{X}(\mathrm{k})|\right)$. 


\section{Statistical Measures and Analysis}

A set of 38 samples (a group of 19 Negative cases and another group of 19 positive cases) are used in this part of our study. The adopted cases are of mixed sex (i.e., male and female). Ages of the studied cases are also varying (with averages ranging from 16 to 73 years), where for any certain case, the ages of the patients from the two COVID related groups are not exactly the same but are selected to be close and of similar age group.

In order to make the most proper final decision of the disease diagnosis and to differentiate between negative and positive cases, selected features; namely, Zero Crossing Rate (ZCR), signal energy, skewness and kurtosis are adopted, measured and analyzed in our approach.

Zero crossing rate indicates the number of times the signal crosses the zero value which basically corresponds to the audio frequency of the cough signal. Figure 8 demonstrates the zero crossing rates for both negative (ZCR_N) and positive cases (ZCR_P), which are plotted for the adopted samples starting with the lower age. We can notice from this figure, that the majority of positive Covid19 cases indicate high ZCR compared with the negative COVID-19 cases. The average value of ZCR of negative cases is ZCR_N = 931.2631579, while the average value of ZCR of positive cases is ZCR_P $=1003.052632$.

The other valuable factor used in this part of our study is the Energy measure. In this measure, energy corresponding to the approximation and energy corresponding to the details, are considered.

Energy corresponding to the approximation for both negative (Eapp_N) and positive cases (Eapp_P) are shown in Fig. 9. This figure shows that the majority of positive COVID-19 cases indicate high energy corresponding to the approximation compared with the negative COVID-19 cases. Some rare cases appeared with the negative cases in which the result seem to be similar to that occurs with the positive cases, indicate that COVID-19 share many similar symptoms with many other diseases. The average value of energy corresponding to the approximation of negative cases is lower than that of the positive cases $\left(\right.$ Epp_N $\mathrm{N}_{\text {avg }}=70.329$ and Epp_P $\left.{ }_{\text {avg }}=75.217\right)$.

The energy corresponding to the details for first, second, third and fourth energy elements are shown in Fig. 10. The average energy values corresponding to the details are tabulated in Table 1.

Looking at the graphs of Fig. 10 and the values of Table 1 , we can conclude that negative cases are demonstrating higher values with respect to those of positive cases.

The factors, Skewness and Kurtosis are also calculated based on Eq. (6) and (7). Their behavior is visualized in Fig. (11a and 11b), respectively. The average values for both factors are shown Table 2 .

Examination of the graphs of Fig. 11 and the values of Table 2, indicate a small difference between the values corresponding to the negative cases with respect to those of positive cases. Therefore, we may conclude that these two factors may not be effective enough to help in the classification and final decision making.

\section{Application of Recurrent Neural Network (RNN)}

Recurrent Neural Network (RNN) is used with an architecture which is composed of sequence input layer, Long Short-Term Memory (LSTM) layer, fully connected layer and regression layer in order to achieve an effective way in this situation (Sherstinsky, 2020).

The proposed approach of the system has relied on the positive and negative data as training set and the average of them as test set with Adam optimizer and 1000 epoch numbers as shown in Fig. 12. The simulated results indicated that the recognition rate of the positive cases is $100 \%$. On the other hand, recognition rate is $90 \%$ for the negative cases because these cases also have some respiratory diseases that leading to false results.

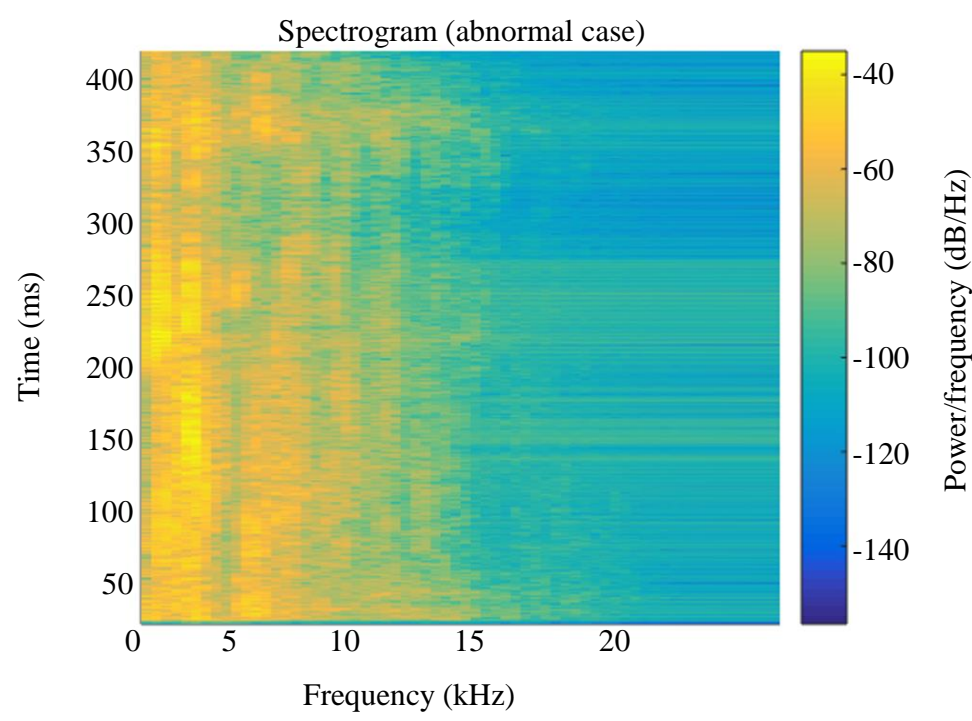

(a) 


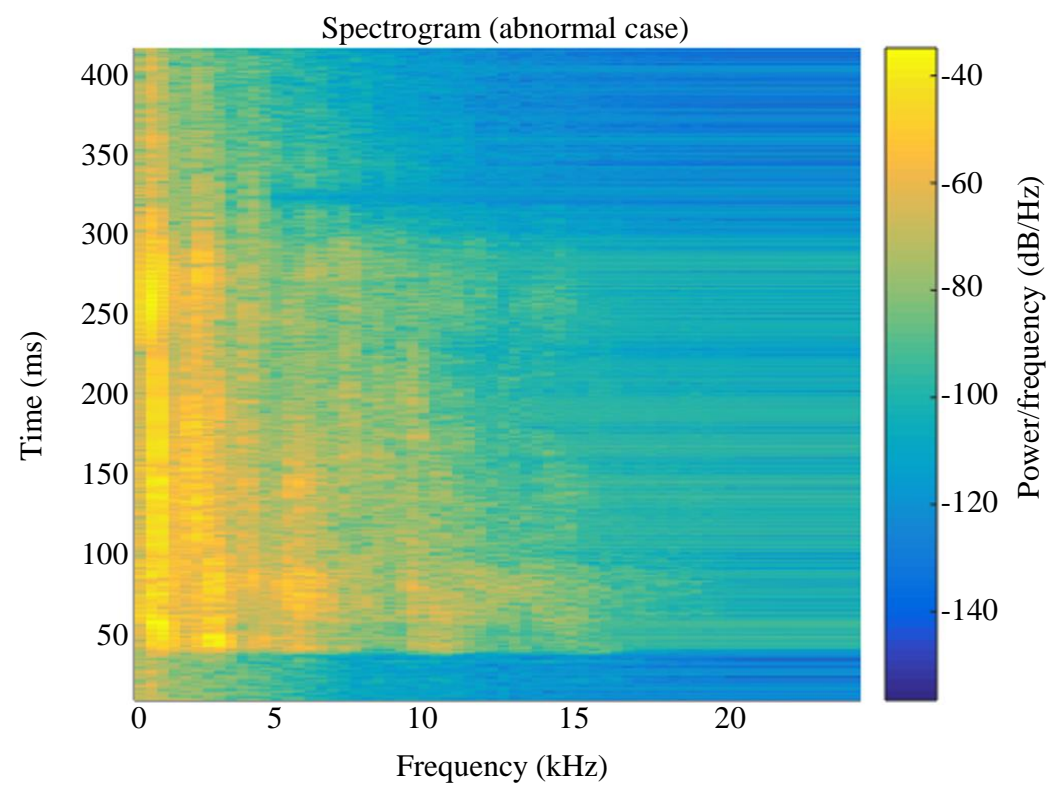

(b)

Fig. 7: Frame spectrogram of cough sound signals (a) Negative case (non-COVID19); (b) Positive case (COVID-19)

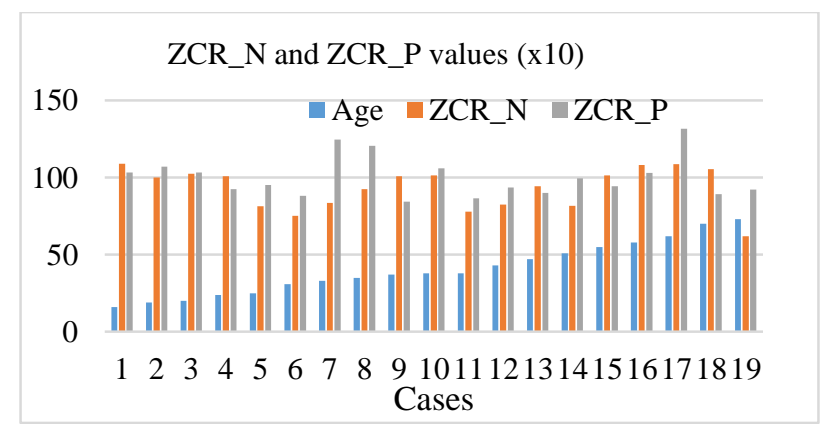

Fig. 8: Zero Crossing Rates (ZCR)

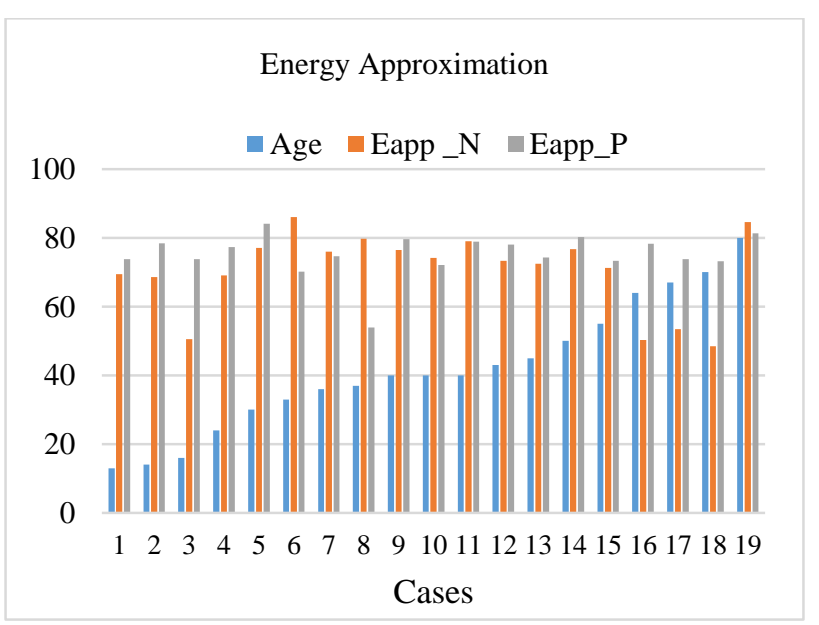

Fig. 9: Energy corresponding to the approximation 


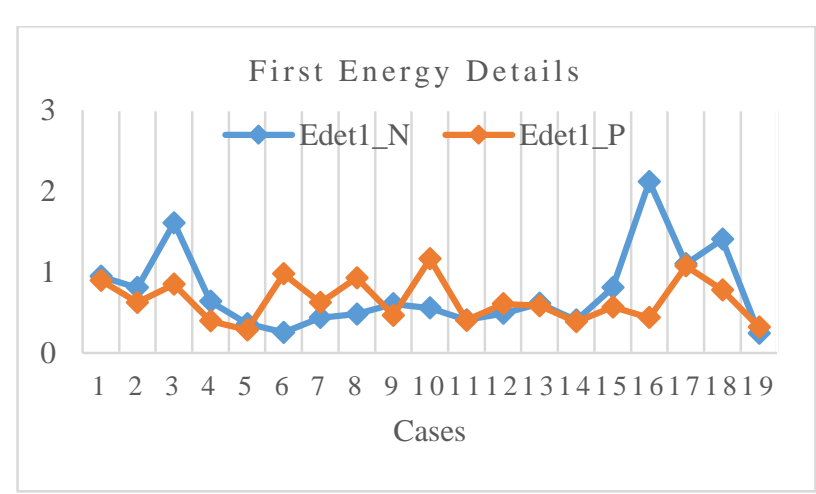

(a)

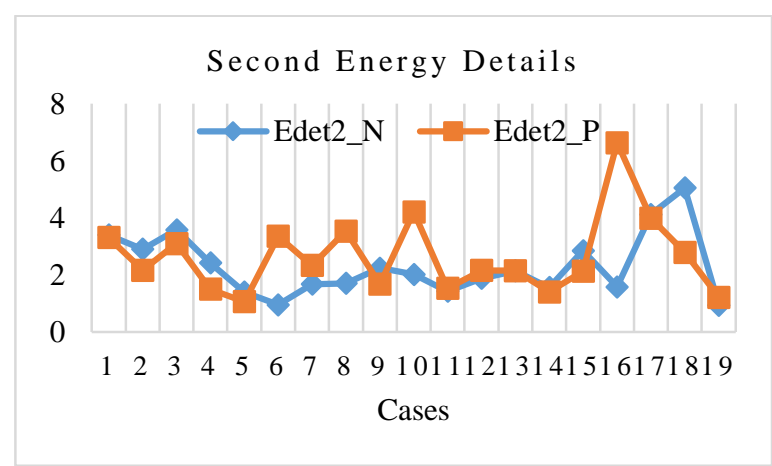

(b)

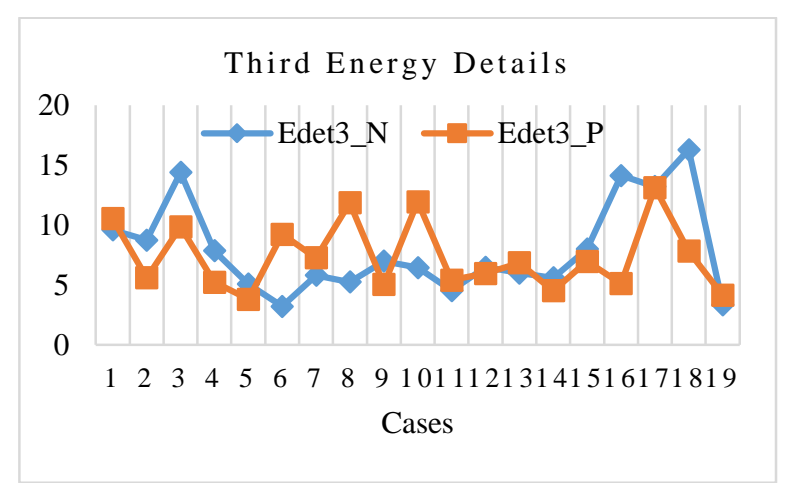

(c)

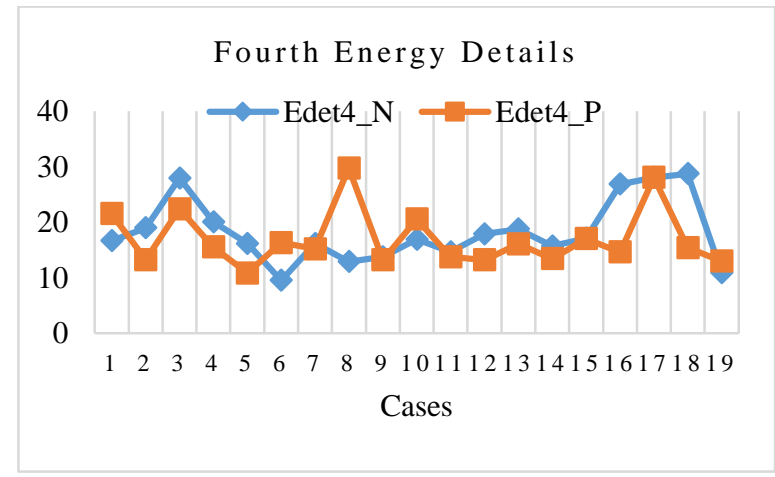

(d)

Fig. 10: Energy corresponding to the details (a) first energy details; (b) second energy details; (c) third energy details; (d) fourth energy details 


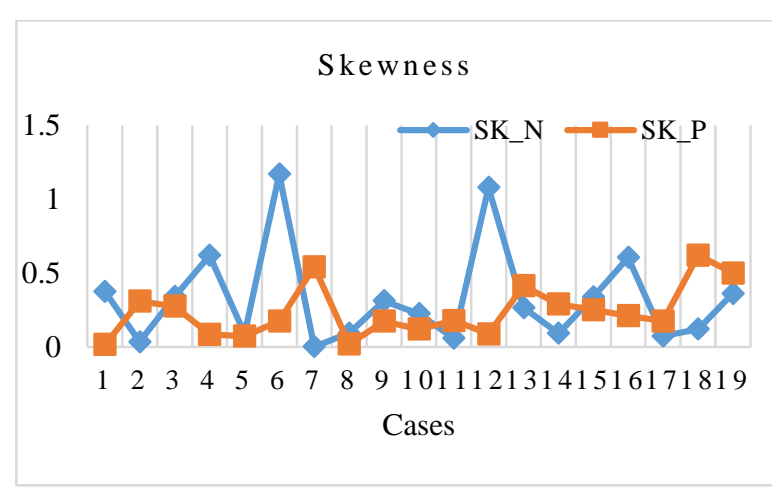

(a)

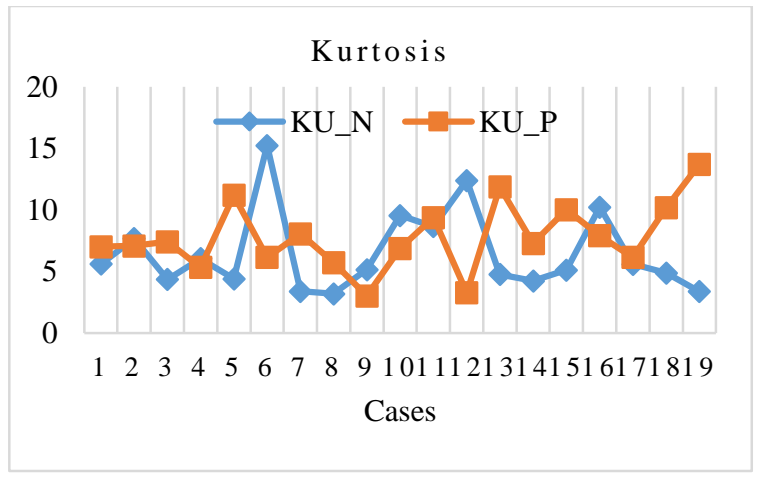

(b)

Fig. 11: Skewness and Kurtosis (a) Skewness; (b) Kurtosis

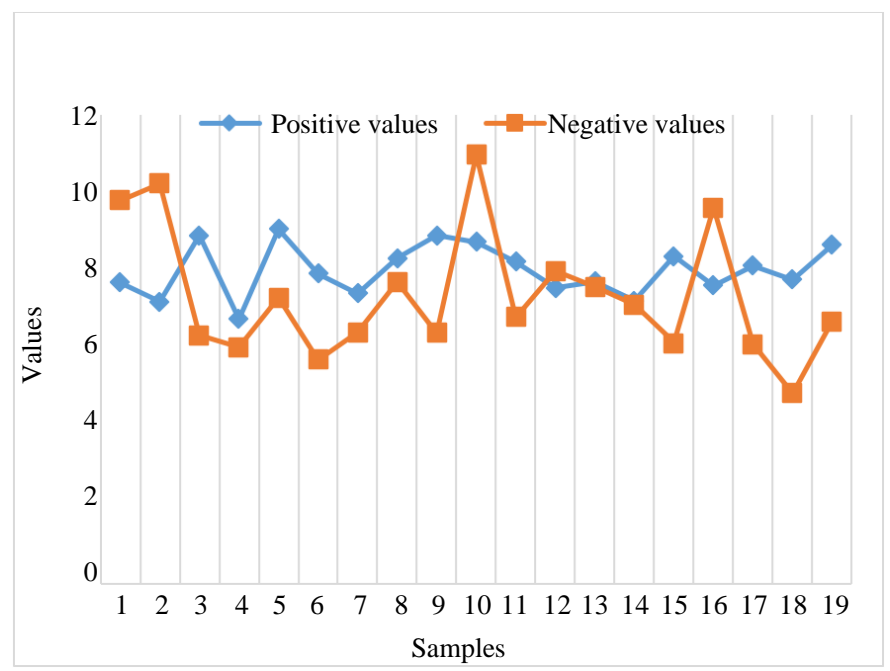

Fig. 12: The Simulated results of RNN application

Table 1: the average energy values corresponding to the details

\begin{tabular}{lcc} 
& Average values (Edet) & \\
Energy Elements & - & \\
\hline First & Negative cases (non-COVID-19) (Edetn_N) & Positive cases (COVID-19) (Edetn_P) \\
Second & 0.751831579 & 0.650852632 \\
Third & 2.679815789 & 2.381715789 \\
Fourth & 7.934563158 & 7.368257895 \\
\hline
\end{tabular}


Table 2: The average values of Skewness and Kurtosis

\begin{tabular}{lll} 
& Average values & \\
Factors & Negative cases (non-COVID-19) (Edetn_N) & Positive cases (COVID-19) (Edetn_P) \\
\hline Skewness & 0.329557895 & 0.238168421 \\
Kurtosis & 6.512363158 & 7.763257895 \\
\hline
\end{tabular}

\section{Conclusion}

Human life has gone through many pandemics, including the coronavirus, which has appeared many times, until the new virus appeared as COVID-19. The detection and diagnosis of the virus in the early stages has the greatest benefit in treatment and limiting the spread of the disease. Automatic detection of COVID-19 disease is an important issue in the management of virus spreading. To minimize the damage caused by various respiratory diseases, it is necessary to develop an approach for analyzing the acquired data from the patient. This research relied on the detection of the virus through coughing, where an approach was designed based on the distribution of energy in the received cough voice samples to verify whether or not the person was infected. The recognition rates are 100 and 90\% for positive cases and negative cases respectively.

Due to the relatively limited number of volunteer patients participated in sampling acquisition, the size of the produced database is not that large, however it is found valuable and effective to produce leading results on our research.

\section{Author's Contributions}

\section{Muzhir Shaban Al-Ani:}

- Make considerable contributions to the conception and design of study

- Applying computing techniques for experimentation and data analysis

- Contributed substantially in interpretation of data and result discussion

- Make significant contribution to drafting the manuscript

- Shares the team extensively to drafting the manuscript critically for important intellectual content

- Help significantly in the approval of the version of the manuscript to be published

- Agreed to be accountable for all aspects of the work in terms of accuracy and integrity

\section{Thabit Sultan Mohammed:}

- Make considerable contributions to the conception and design of study

- Contributed substantially in interpretation of data and result discussion
- Make significant contribution to drafting the manuscript

- Shares the team extensively to drafting the manuscript critically for important intellectual content

- Help significantly in the approval of the version of the manuscript to be published

- Agreed to be accountable for all aspects of the work in terms of accuracy and integrity

\section{Hasan Ismail Sultan:}

- Make considerable contributions to the conception and design of study

- Participated with a considerable contribution to acquisition of data

- Contributed substantially in interpretation of data and result discussion

- Make significant contribution to drafting the manuscript

- Shares the team extensively to drafting the manuscript critically for important intellectual content

- Help significantly in the approval of the version of the manuscript to be published

- Agreed to be accountable for all aspects of the work in terms of accuracy and integrity

\section{Khattab M. Ali Alheeti:}

- Applying computing techniques for experimentation and data analysis

- Contributed substantially in interpretation of data and result discussion

- Make significant contribution to drafting the manuscript

- Shares the team extensively to drafting the manuscript critically for important intellectual content

- Help significantly in the approval of the version of the manuscript to be published

- Agreed to be accountable for all aspects of the work in terms of accuracy and integrity

\section{Karim Mohammed Aljebory:}

- Contributed substantially in interpretation of data and result discussion

- Make significant contribution to drafting the manuscript 
- Shares the team extensively to drafting the manuscript critically for important intellectual content

- Help significantly in the approval of the version of the manuscript to be published

- Agreed to be accountable for all aspects of the work in terms of accuracy and integrity

\section{Awni Ismail Sultan:}

- Participated with a considerable contribution to acquisition of data

- Make significant contribution to drafting the manuscript

- Shares the team extensively to drafting the manuscript critically for important intellectual content

- Help significantly in the approval of the version of the manuscript to be published

- $\quad$ Agreed to be accountable for all aspects of the work in terms of accuracy and integrity

\section{Ethics}

This article is original and contains unpublished material. The corresponding author confirms that all of the other authors have read and approved the manuscript and no ethical issues involved.

\section{References}

Abeyratne, U. R., Swarnkar, V., Setyati, A., \& Triasih, R. (2013). Cough sound analysis can rapidly diagnose childhood pneumonia. Annals of Biomedical Engineering, 41(11), 2448-2462. https://doi.org/10.1109/EMBC.2013.6610724

Agyeman, A. A., Chin, K. L., Landersdorfer, C. B., Liew, D., \& Ofori-Asenso, R. (2020, August). Smell and taste dysfunction in patients with COVID-19: a systematic review and meta-analysis. In Mayo Clinic Proceedings (Vol. 95, No. 8, pp. 1621-1631). Elsevier. https://doi.org/10.1016/j.mayocp.2020.05.030

Al-Ani, M. S. (2017). Study the characteristics of finite impulse response filter based on modified Kaiser window. UHD Journal of Science and Technology, 1(2), 1-6. https://doi.org/10.21928/uhdjst.v1n2y2017.pp1-6

Alheeti, K. M. A., Awad, A. M., \& Al-Ani, M. S. (2019, October). ECG Waveform Encryption Using Shifted FFT and DWT. In 2019 12th International Conference on Developments in eSystems Engineering (DeSE) (pp. 351-356). IEEE. https://doi.org/10.1109/DeSE.2019.00071

Al-Shayea, Q., \& Al-Ani, M. (2016). Efficient window approach of FIR filter design (MSK2). IJCSNS International Journal of Computer Science and Network Security, 16(2), 63-68. http://paper.ijcsns.org/07_book/201602/20160211.pdf
Barry, S. J., Dane, A. D., Morice, A. H., \& Walmsley, A. D. (2006). The automatic recognition and counting of cough. Cough, 2(1), 1-9. https://doi.org/10.1186/1745-9974-2-8

Brown, C., Chauhan, J., Grammenos, A., Han, J., Hasthanasombat, A., Spathis, D., ... \& Mascolo, C. (2020, August). Exploring automatic diagnosis of covid-19 from crowdsourced respiratory sound data. In Proceedings of the 26th ACM SIGKDD International Conference on Knowledge Discovery and Data Mining (pp. 3474-3484). https://doi.org/10.1145/3394486.3412865

Chatrzarrin, H., Arcelus, A., Goubran, R., \& Knoefel, F. (2011, May). Feature extraction for the differentiation of dry and wet cough sounds. In 2011 IEEE International Symposium on Medical Measurements and Applications (pp. 162-166). IEEE. https://doi.org/10.1109/MeMeA.2011.5966670

Cheng, M. P., Papenburg, J., Desjardins, M., Kanjilal, S., Quach, C., Libman, M., ... \& Yansouni, C. P. (2020). Diagnostic testing for severe acute respiratory syndrome-related coronavirus 2: a narrative review. Annals of Internal Medicine, 172(11), 726-734. https://doi.org/10.7326/M20-1301

DeCarlo, L. T. (1997). On the meaning and use of kurtosis. Psychological Methods, 2(3), 292. https://doi.org/10.1037/1082-989X.2.3.292

Derraz, M. (2020). Remotely Diagnose Coronavirus by Recognizing and Counting of Coughs During Phone Calls. Biomedical Research and Reviews, vol.3(3);125, pp,1-8. https://doi.org/10.31021/brr.20203125

Hoang, M. P., Kanjanaumporn, J., Aeumjaturapat, S., Chusakul, S., Seresirikachorn, K., \& Snidvongs, K. (2020). Olfactory and gustatory dysfunctions in COVID-19 patients: a systematic review and meta-analysis. Asian Pac Journal Allergy Immunol, 38(3), 162-169. https://doi.org/10.12932/AP-210520-0853.

Holland, M., Zaloga, D. J., \& Friderici, C. S. (2020). COVID-19 Personal Protective Equipment (PPE) for the emergency physician. Visual Journal of Emergency Medicine, 19, 100740. https://doi.org/10.1016/j.visj.2020.100740

Huang, C., Wang, Y., Li, X., Ren, L., Zhao, J., Hu, Y., ... \& Cao, B. (2020). Clinical features of patients infected with 2019 novel coronavirus in Wuhan, China. The Lancet, 395(10223), 497-506. https://doi.org/10.1016/S0140-6736(20)30183-5.

HuBer, J., \& Stathopoulos, E. T. (2015). 2 Speech Breathing Across the Life Span and in Disease. The Handbook of Speech Production, 13 https://doi.org/10.1002/9781118584156.ch2 
Imran, A., Posokhova, I., Qureshi, H. N., Masood, U., Riaz, M. S., Ali, K., ... \& Nabeel, M. (2020). AI4COVID-19: AI enabled preliminary diagnosis for COVID-19 from cough samples via an app. Informatics in Medicine Unlocked, 20, 100378. https://doi.org/10.1016/j.imu.2020.100378

Infante, C., Chamberlain, D., Fletcher, R., Thorat, Y., \& Kodgule, R. (2017, October). Use of cough sounds for diagnosis and screening of pulmonary disease. In 2017 IEEE Global Humanitarian Technology Conference (GHTC) (pp. 1-10). IEEE. https://doi.org/10.1109/GHTC.2017.8239338

Mehraeen, E., Behnezhad, F., Salehi, M. A., Noori, T., Harandi, H., \& SeyedAlinaghi, S. (2020). Olfactory and gustatory dysfunctions due to the coronavirus disease (COVID-19): a review of current evidence. European Archives of Oto-Rhino-Laryngology, 1-6. https://doi.org/10.1007/s00405-020-06120-6.

Miranda, I. D., Diacon, A. H., \& Niesler, T. R. (2019, July). A comparative study of features for acoustic cough detection using deep architectures. In 2019 41st Annual International Conference of the IEEE Engineering in Medicine and Biology Society (EMBC) (pp. 2601-2605). IEEE. https://doi.org/10.1109/embc.2019.8856412

Mohammed, T. S., Rasheed, M., Al-Ani, M., Al-Shayea, Q., \& Alnaimi, F. (2020). Fault diagnosis of rotating machine based on audio signal recognition system: an efficient approach. International Journal Simulation System Science Technology, https://ijssst.info/Vol-21/No-1/paper8.pdf

Ni, W., Yang, X., Yang, D., Bao, J., Li, R., Xiao, Y., ... \& Gao, Z. (2020). Role of angiotensin-converting enzyme 2 (ACE2) in COVID-19. Critical Care, 24(1), 1-10. https://doi.org/10.1186/s13054-020-03120-0.

PAHO/WHO. (2020). Epidemiological Alert Complications and sequelae of COVID-19. American Health Organization/World Health Organization 12 August. pp, 1-16. URL: https://www.paho.org/en/documents/epidemiologica 1-alert-complications-and-sequelae-COVID-19-12august-2020.

Porter, P., Abeyratne, U., Swarnkar, V., Tan, J., Ng, T. W., Brisbane, J. M., ... \& Della, P. (2019). A prospective multicentre study testing the diagnostic accuracy of an automated cough sound centred analytic system for the identification of common respiratory disorders in children. Respiratory Research, 20(1), 1-10. https://doi.org/10.1186/s12931-019-1046-6

Pramono, R. X. A., Imtiaz, S. A., \& Rodriguez-Villegas, E. (2019, July). Automatic cough detection in acoustic signal using spectral features. In 2019 41st Annual International Conference of the IEEE Engineering in Medicine and Biology Society (EMBC) (pp. 7153-7156). IEEE. https://doi.org/10.1109/embc.2019.8857792
Rocchesso, D. (2003). Introduction to sound processing. Mondo estremo. https://www.researchgate.net/deref/http $\% 3 \mathrm{~A} \% 2 \mathrm{~F} \% 2$ Fdx.doi.org\%2F10.2307\%2F3680990

Rudraraju, G., Palreddy, S., Mamidgi, B., Sripada, N. R., Sai, Y. P., Vodnala, N. K., \& Haranath, S. P. (2020). Cough sound analysis and objective correlation with spirometry and clinical diagnosis. Informatics in Medicine Unlocked, 19, 100319. https://doi.org/10.1016/j.imu.2020.100319

Sharma, N., Krishnan, P., Kumar, R., Ramoji, S., Chetupalli, S. R., Ghosh, P. K., \& Ganapathy, S. (2020). Coswara--A Database of Breathing, Cough and Voice Sounds for COVID-19 Diagnosis. arXiv Preprint arXiv:2005.10548. ttps://doi.org/10.21437/Interspeech.2020-2768.

Sheridan, C. (2020). Fast, portable tests come online to curb coronavirus pandemic. Nat Biotechnol, 38(5), 515-518. https://doi.org/10.1038/d41587-020-00010-2

Sherstinsky, A. (2020). Fundamentals of recurrent neural network (RNN) and long short-term memory (LSTM) network. Physica D: Nonlinear Phenomena, 404, 132306. https://doi.org/10.1016/j.physd.2019.132306

Shi, Y., Liu, H., Wang, Y., Cai, M., \& Xu, W. (2018). Theory and application of audio-based assessment of cough. Journal of Sensors, 2018. https://doi.org/10.1155/2018/9845321

Shi, Y., Wang, G., Cai, X., Deng, J., Zheng, L., Zhu, H., Zheng, M., Yang, B., \& Chen, Z. (2020). An Overview of COVID-19", Journal of Zhejiang UniversityScience B (Biomedicine and Biotechnology), May, pp, 343-360. https://doi.org/10.1631/jzus.B2000083

Soliński, M., Łepek, M., \& Kołtowski, Ł. (2020). Automatic cough detection based on airflow signals for portable spirometry system. Informatics in Medicine Unlocked, 18, 100313. https://doi.org/10.1016/j.imu.2020.100313

Song, I. (2015, July). Diagnosis of pneumonia from sounds collected using low cost cell phones. In 2015 International joint conference on neural networks (IJCNN) (pp. 1-8). IEEE. https://doi.org/10.1109/IJCNN.2015.7280317.

Sultan, H., Sultan, A., \& Maded, Z. (2020). A descriptive study of COVID-19 infection among symptomatic patients in Al- Hawija districtKirkuk- Iraq. International Journal of Psychosocial Rehabilitation. 24, pp, 9622-9629. https://doi.org/10.37200/IJPR/V24I10/PR300435

Tabata, S., Imai, K., Kawano, S., Ikeda, M., Kodama, T., Miyoshi, K., ... \& Tamura, K. (2020). Clinical characteristics of COVID-19 in 104 people with SARS-CoV-2 infection on the Diamond Princess cruise ship: a retrospective analysis. The Lancet Infectious Diseases, 20(9), 1043-1050. https://doi.org/10.1016/S1473-3099(20)30482-5 
Thorpe, W., Kurver, M., King, G., \& Salome, C. (2001, November). Acoustic analysis of cough. In The Seventh Australian and New Zealand Intelligent Information Systems Conference, 2001 (pp, 391-394). IEEE. https://ieeexplore.ieee.org/abstract/document/974110/

Velavan, T. P., \& Meyer, C. G. (2020). Mild versus severe COVID-19: laboratory markers. International Journal of Infectious Diseases, 95, 304-307. https://doi.org/10.1016/j.ijid.2020.04.061

Wali, M. K., Murugappan, M., Ahmad, R. B., \& Zheng, B. S. (2012, February). Development of discrete wavelet transform (DWT) toolbox for signal processing applications. In 2012 International Conference on Biomedical Engineering (ICoBE) (pp. 211-216). IEEE. https://ieeexplore.ieee.org/abstract/document/6179007/

Wang, D., Hu, B., Hu, C., Zhu, F., Liu, X., \& Zhang, J. (2020). Clinical Characteristics of 138 Hospitalized Patients With 2019 Novel Coronavirus-Infected. Pneumonia in Wuhan, China. JAMA. 323, pp. 1061-1069. https://doi.org/10.1001/jama.2020.1585

WHO. (2020a). Advice on the use of masks in the context of COVID-19: interim guidance, 5 June 2020 (No. WHO/2019-nCoV/IPC_Masks/2020.4). World Health Organization. https://doi.org/10.15557/PiMR.2020.0005
WHO. (2020b). Coronavirus disease 2019 (COVID-19): situation report, 82. https://apps.who.int/iris/handle/10665/331865

Xavier, A. R., Silva, J. S., Almeida, J. P. C., Conceição, J. F. F., Lacerda, G. S., \& Kanaan, S. (2020). COVID-19: clinical and laboratory manifestations in novel coronavirus infection. Jornal Brasileiro de Patologia e Medicina Laboratorial, 56. https://doi.org/10.5935/1676-2444.20200049

Xu, M., Wang, D., Wang, H., Zhang, X., Liang, T., Dai, J., ... \& Yu, X. (2020). COVID-19 diagnostic testing: Technology perspective. Clinical and Translational Medicine, 10(4), e158. https://dx.doi.org/10.1002\%2Fctm2.158

You, M., Wang, H., Liu, Z., Chen, C., Liu, J., Xu, X. H., \& Qiu, Z. M. (2017). Novel feature extraction method for cough detection using NMF. IET Signal Processing, 11(5), 515-520. https://doi.org/10.1049/iet-spr.2016.0341

Young, D., Tatarian, L., Mujtaba, G., Chow, P., Ibrahim, S., \& Joshi, G. (2020). Chest CT versus RT-PCR for diagnostic accuracy of COVID-19 detection: A meta-analysis. Journal Vasc Medicine Surg, 8(3). https://doi.org/10.35248/2329-6925.20.8.392 